\title{
SYNTHESIS AND CHARACTERIZATION OF RARE-EARTH NANOPARTICLES.
}

\author{
J. A. Ascencio and G. Canizal. \\ Programa de Investigación y Desarrollo de Ductos. Instituto Mexicano del Petróleo, \\ Lázaro Cárdenas 152, San Bartolo Atepehuacán, C.P. 07730, México, D. F., México.
}

Nanotechnology as a reality involves many areas; however the most important is to be able of making materials design focusing efforts to a specific application. The use of metals in different fields as medicine is well known and vastly studied from point of views really different as technologies of metal removal from water or soils until the design of molecular systems to produce materials with specific capacities. Three main parameters must be always considered: size and elemental compositions, which involve different effects in the macroscopic properties and the local chemical selectivity. In this work, the synthesis by bioreduction methods and characterization using high resolution transmission electron microscopy and atomic force microscopy, for the case rare earth nanoparticles is reported and the application of them is focused to the use of these clusters in nanomedicine, where the application of samarium, and ytterbium can be identified because they involve radiation potential involved in their nature. So then the molecular design of clusters of lanthanide elements is oriented to nuclear nanomedicine by using a specific number of source radiation atoms. Evidences of controlled size production of nanoparticles will be shown and a general scope of the efforts is also planned to be presented. In the figure 1, two different images of samarium clusters are shown, one of them corresponds to bare particles and the second one shows a group of nanoparticles covered by the biomass that is used in the bio-reduction. In the second figure, a HREM image for bigger arrays of nanoparticles is shown. Small particles can be clearly identified in the border of the substrate, wit sizes of around $2 \mathrm{~nm}$ which allows to be used in much kind of applications from nanomedicine to many other technologies. 
$\underline{\mathbf{a}}$

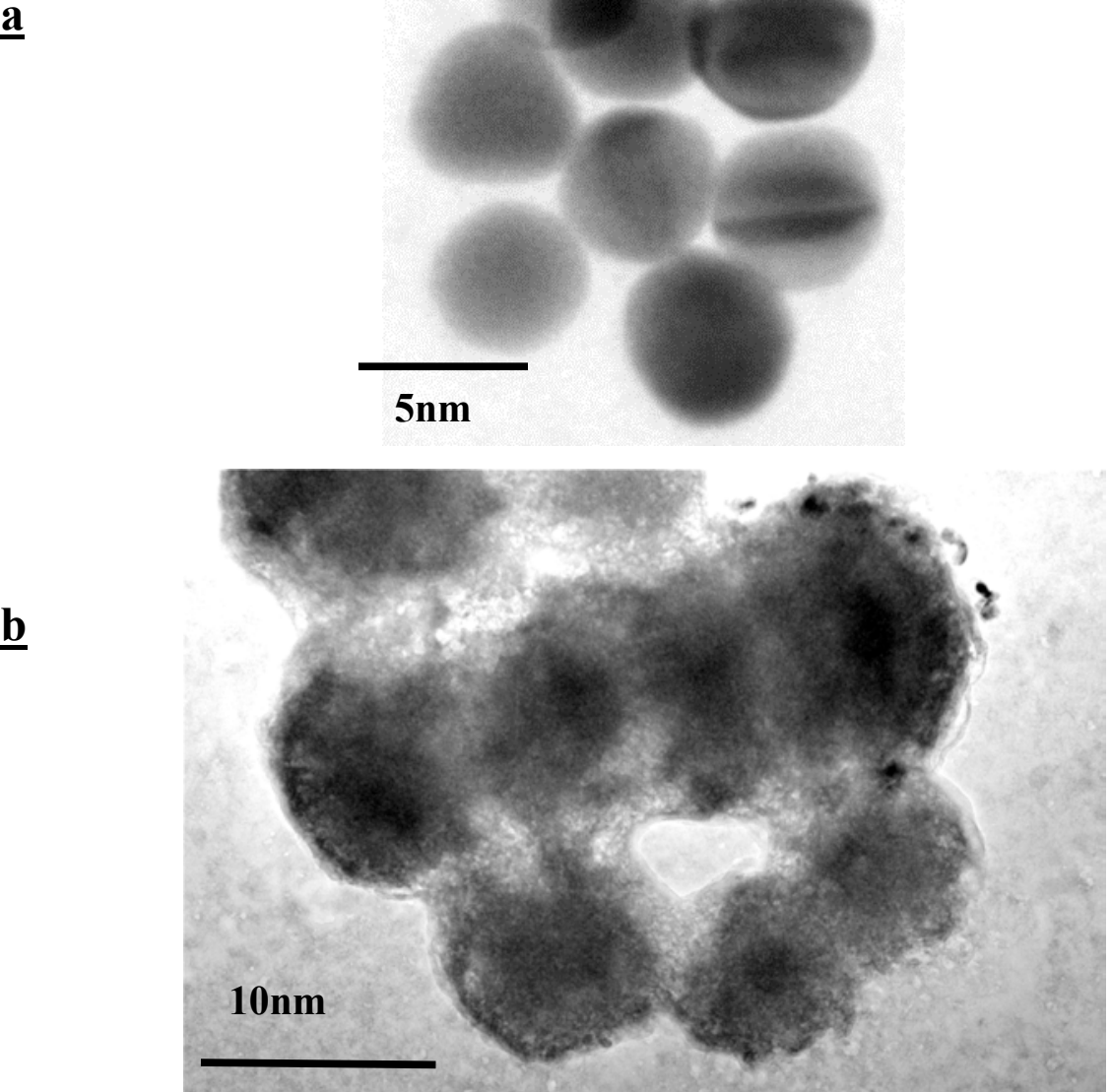

Figure 1. Nanoparticles of Samarium a) bare and b) covered by the biomass. An electron diffraction pattern obtained of one of the clusters is also shown in c).

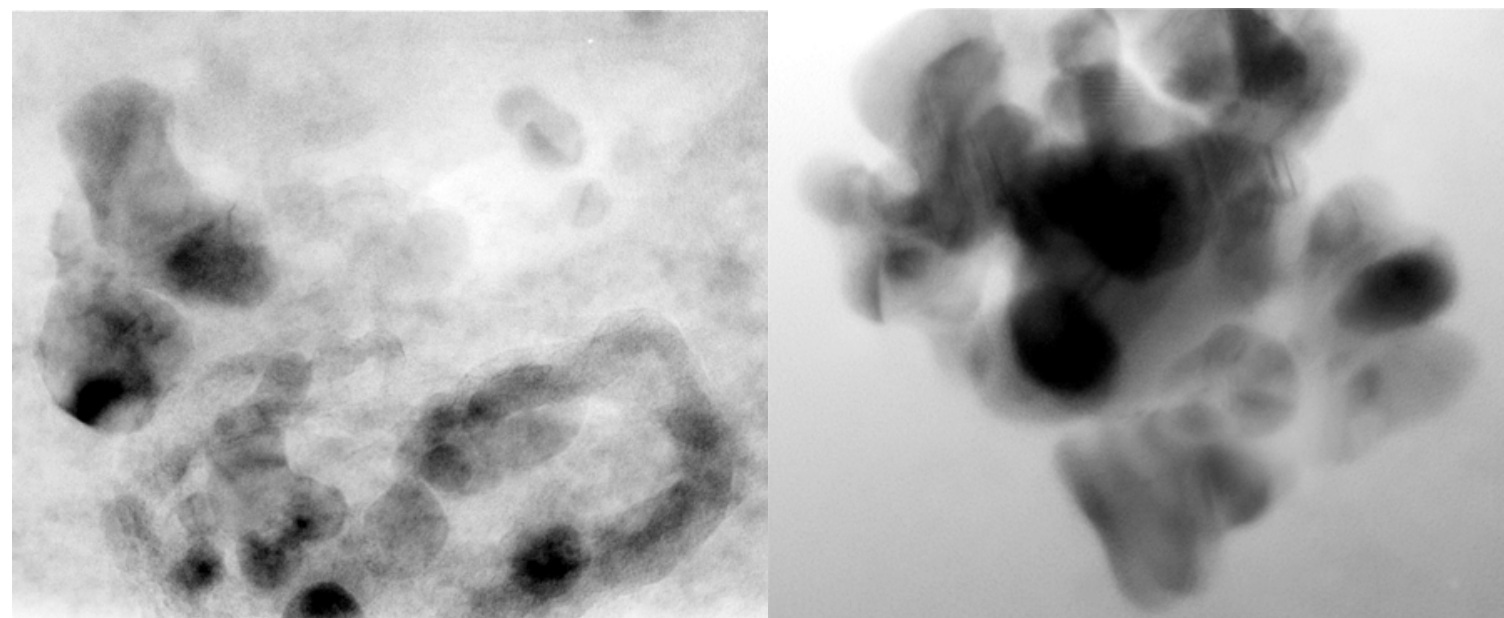

Figure 2. Nanoparticles of samarium in a amorphous carbon matrix. 